\title{
3D Object Recognition Based on Feature Line Extracting
}

\author{
Zhijia Zhang, Boshi Wang \\ Shenyang University of Technology \\ Shenyang. China \\ E-mail:zzjsut@126.com
}

\author{
Wenqiang Li, Dan Zhang \\ Shenyang University of Technology \\ Shenyang.China \\ E-mail:liwenqiang1217@gmail.com
}

\begin{abstract}
For many practical applications in industrial and medical fields, 3D object recognition based on feature extracting has become an actively investigative field. In general, 3D object recognition system can be completed through two stages. Firstly, we use 3D reconstruction of input data to get object expression. Then we use 3D invariant to identify objects. In order to promote further development of this field, this paper summarizes the 3D object recognition technology research, and introduces typical methods of modern 3D object recognition, the concepts of 3D reconstruction, 3D invariants. In the process of 3D object recognition, feature line extraction, 3D invariant calculation, matching strategy, 3D object recognition fields are reviewed. The first section presents the significance of $3 \mathrm{D}$ object recognition. The second section presents the development of object recognition. The third section presents the double viewpoint of $3 \mathrm{D}$ reconstruction. The fourth section presents the 3D reconstruction of image processing. The fifth section presents the 3D invariant technology. The sixth section presents the problems and development direction.
\end{abstract}

Keywords-image processing; feature line extraction; $3 D$ reconstruction; $3 D$ invariant; matching strategy ; $3 D$ object recognition

\section{SIGNIFICANCE OF 3D OBJECT RECOGNITION}

Machine vision is a new method to replace human observation and understanding of the world. Machine vision by using computer technology and optical means to gain a vice or many images taken by the 3D objects reduction shape, get $3 \mathrm{D}$ data value, and the ability to restore a $3 \mathrm{D}$ object $3 \mathrm{D}$ coordinates. It is the essence of the $3 \mathrm{D}$ object to perceive the 2D image study extracts 3D scene world physical process. For all kinds of target image can obtain, can use the machine vision measurement of the related technologies to get his shape, size and motion parameters[1]. As industry and medical care and other fields of application requirement of reality has increased dramatically, 3D object recognition technology has become a very active field of research, it is widely used in automatic navigation, automatic detection, assembly tasks and the medical image analysis system. 3D object recognition especially of modern industrial automation production can be involved in all kinds of objects in production test and 3D topography detection.

\section{The Development Of 3D OBJect Recognition}

3D object recognition technology development benefits from the improvement of machine vision. It has proposed a lot of different frames and the methods to solve the problem of object recognition.

\section{A. Geometrical Characteristics-Based Object Recognition}

The method which based on the object recognition of geometric features extracted the gauss curvature, mean curvature, curvature histogram, curvature of entropy [2] from the depth images in the scene. Describe objects with a property relationship chart ARG and match ARG figure with the model in model library to identify objects.

\section{B. 3D Object Recognition of Curvature Affine Invariant}

Curvature affine invariant method proposes a 3D object recognition of the approximate plane rapid method. The method use the curvature of the construction information to outline the affine invariant function, and use the invariant extreme value point to get information for similarity comparison realizing the target recognition [3].

\section{3D Object Recognition Algorithm of Morphology}

After establishing the identity model based on the objects, we could find out all of the object shape charts and features, and take out their topology information and geometry information. Next, contour extraction and track boundary of object would get frame diagram of 2D images, and take out their topology information and geometry information. Finally, match topology information and geometry information to achieve the purpose of recognition.

\section{3D Invariant Object Recognition Technology}

3D object recognition based on the 3D invariant technology is a very important research in recent years. 3D object recognition technology is a new 3D target recognition technology accompanied by becoming mature of geometric invariants and 3D object recognition technology[4]. It evolved from the traditional 2D image recognition method. In view of the flexibility of $2 \mathrm{D}$ projective transform solution, we can use different solution of setting elements. Although, the calculation method presents $1 \mathrm{D}$ or $2 \mathrm{D}$ form, its essence reflects relationship of 3D space geometrical element. In object recognition, the 3D geometric invariable method which use target recognition, feature point and solution of grid recognition has been improved. The identification precision rate of this method is high, it can effectively identify some simple structure of 3D objects. In addition, it also has characteristics, such as simple modeling and convenient operation[5]. Compared with the traditional 3D object recognition technology, it has the advantages of high sensitivity rate, so its application has a broad prospect.

\section{DOUBLE VIEWPOINT OF 3D RECONSTRUCTION}

Binocular stereo vision is based on the principle of the 3D information acquisition. According to the relationship between 
the positions of two cameras, we can get 3D objects size and 3D space coordinate of feature points. Binocular stereo vision system is generally consisted by two cameras or one moving camera. 3D measurement system's hardware has directly effect on measurement precision of the system. Unreasonable hardware can not only reduce the stability of the system, but also drop the anti-interference ability, and increase the hardware cost. These may affect system calculative speed and accuracy of measurement. So, it is necessary to choose right components for forming a stable and the reasonable allocation of hardware environment.

$3 \mathrm{D}$ reconstruction is the inverse of the projection process, and determines spatial point through the projection point. According to consider projection point and camera attachment point of the projection equation, it would get two images to find out the points at least[6]. This process is called triangle measuring. So it will get a matching point to complete the $3 \mathrm{D}$ reconstruction. The method of $3 \mathrm{D}$ matching has sift method and affine invariant method, but any kind of image matching may have mistakes. This paper combines man-computer interactive method with sift matching algorithm method. This improved method greatly enhances the accuracy of the matching, providing a good foundation for 3D reconstruction. As shown in figure 1.

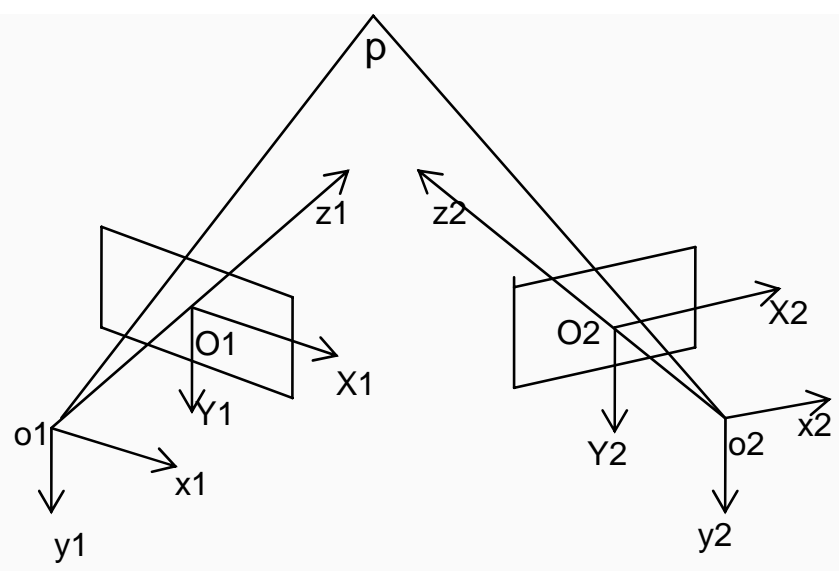

Figure 1 Double viewpoint of 3D reconstruction

\section{A. View Data Comprehensive Optimization}

To get a completed 3D object model, it has to carry on the comprehensive view data. The coordinates of synthesis have to solve two questions: the obtaining of two coordinates' movement; the proportion conversion of two coordinates. It needs to calculate the changes of coordinates scale, rotation matrix, translation vector, and corresponding points of two coordinates. Because of the existence of error, the optimal algorithm would be used to design the evaluation function to optimize error. After obtaining two coordinates' changes, all 3D points obtained by double viewpoint can be unified to a designated world coordinate system. When find out the 3D coordinate, we should make their coordinates and position optimized and use 2D and 3D attribute function optimize it. In addition, spatial point neighborhood projection of plane area and plane feature point neighborhood has certain errors, which need to be used by function optimization. Extract local image projective information from optimization function and make the $3 \mathrm{D}$ reconstruction to meet the reality as far as possible.

\section{3D RECONSTRUCTION OF IMAgE PROCESSING}

\section{A. Image Smoothing}

An image may have parasitic effect. These parasitic effect may be produced on the transmission, also may be produced in quantitative processing. The purpose of image smoothing is used to improve the quality of the image sampling, these parasitic effect factors and generation reduce image edge and blurred lines[7]. The image smoothing processing method has the airspace method and the frequency domain method. It includes a neighborhood average method, low pass filtering method and image average method.

\section{B. Image Sharpening}

After smooth filtering, the image of high frequency noise is removed, but it may make the image become blurred. The main purpose of image sharpening is to highlight the image details or enhanced the fuzzy details. It usually has two kinds of methods: differential method and high-pass filter method. The common differential sharpen method includes gradient sharpen method and Laplace sharpen method[8].

\section{Feature Point Extracting}

The extraction of feature points is divided into two categories: One is based on the contour lines method and the other is based on image gray value method. The method that based on contour line firstly extract things from the image edge, then search maximum curvature point at the edge of the chain. At the edge of polygon, it calculates the polygon vertices as a feature point[9]. The method based on the image gray value is defining some operators, extracting characteristic points of extreme value through the gray image for the operator. The process includes calculating each pixel of the Robert gradient, using the differential operators to extract the primary point and calculation of covariance matrix. If a pixel value in the pixel is the center of an appropriate window in the great value, then the pixel is a pixel level feature point.

\section{Feature Line Extracting}

Using canny edge method for image processing, after edge extraction completing, we can get the edge information and feature line extraction. For an image, we can use the following steps to finish feature line extraction. The process includes defining the characteristic line's originating endpoint, searching edge points in a neighborhood, calculating characteristic linear equation, calculating error, searching point set, finding the feature points and recording the feature line.

\section{3D INVARIANT TECHNOLOGY}

The method based on the $3 \mathrm{D}$ invariant object recognition technology is emerged as a new object recognition technology and become mature with invariant and 3D object recognition technology[10]. It is the method evaluated from the traditional $2 \mathrm{D}$ recognition. 3D object recognition identify and locate the object from the 2D image. Because of the shape of object is changing with the view position, angle, target attitude and the other imaging parameters, such changes are sometimes very 
remarkable. These changes may lead to conventional identification methods failure. The method based on the 3D invariant object recognition begins with basic properties of the projective geometry. Using various of points and lines to establish view relationship, and extract multiple 3D invariant method from complex object. Thus, it is necessary to eliminate impacts with the viewpoint position, angle, target attitude and imaging parameters[11]. 3D invariant method can identify any object in any perspective, and using the object reconstruction can extract world coordinate of any point from object surface, calculating invariants with many corresponding points and lines in the space[12,14]. Transform object recognition based on the 3D shape into invariant data matching to achieve object recognition of arbitrary angle. The $3 \mathrm{D}$ object recognition system as shown in figure 2 .

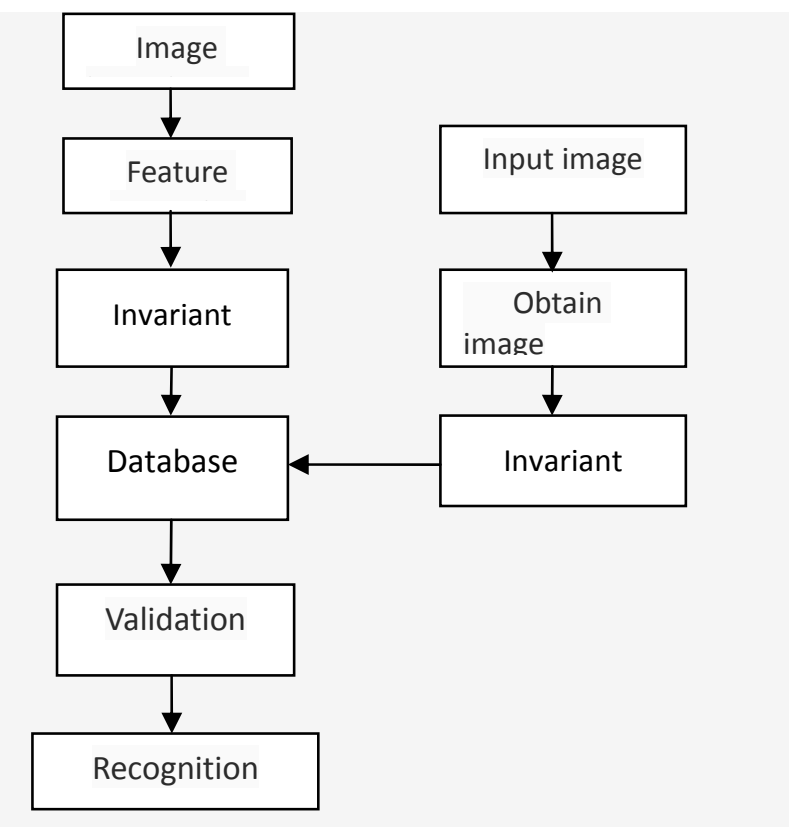

Figure 2 3D object recognition system

\section{A. Recognition Steps of 3D Invariant Object}

Establishing a model recognition storage for the identify objects, record model numbers and object types expression parameters in model library. Use the calculated 3D invariants to establish database index table. Calculate the 3D objects invariants by the input image retrieval models, using the 3D invariants to access database index table, and look for possible matching models. In general, the calculation of the object invariants may be multiple. So when retrieve, we need to vote each of index table and then if there was a vote greater than threshold models, it can be known as a possible matching model[15,16]. Firstly, use image characteristics corresponding relation of model and the part image to calculate 3D model and 2D image projection transformation. The 3D model will be projected onto a $2 \mathrm{D}$ image, calculating matching degree of the model projection and input objects image. If there were more than one certain value in image, consider that the input image objects have been identified.

\section{B. Matching Process of 3D Invariant Object}

According to obtain image expression from 3D object recognition, we need to get identification and positioning of the object in the scene. This recognition is matching the features from the characteristics of object with object model in the scene. Transform the 3D object model matching into data matching, simplifying the matching process, improving the efficiency of the matching[17]. The combination of 3D invariant with hash table is the effective way to improve the efficiency. The reason is that 3D invariance is an unchanging property of object, not depend on observation viewpoint and camera parameters, so it has the good conditions as the index function. Hash table method uses index function to get database records directly. In advance establish the index value for each model, when contrast calculated invariants and index value of each model in search, some possible models can be found.

\section{The PROBlems AND DeVElopMent DiRECTION}

Firstly, the "global and local" contradictory has been existence in 3D object representation method for years. On one hand it is expressed in practical application, object might be only partially visible in the actual situation, so only the object local features can be used. The local feature extraction from scene data cannot describe objects in all shapes, so it is not enough to distinguish the objects; On the other hand, although the global expression can describe the objects better and have a better ability to distinguish them, it cannot be used on an object in a scene blocked, and it may produce a high computation complexity [18].

Secondly, many applications on geometric invariance are needed to use 3D geometric model to obtain the invariant and do hypothesis test. Due to build 3D geometric model of complex objects is difficult and the workload is very large, it is necessary to study the suitable geometric invariance by 2D image object modeling and hypothesis testing method. The traditional 3D modeling method which can exactly describe the $3 \mathrm{D}$ objects is facing the heavy workload. In many feature faces and feature points, local occluded object or complex object is not easy to be described, and the modeling accuracy is not high. If using 2D image to express objects may have unexpected effects, and it can help to describe complex object with many characteristics.

Thirdly, at present the perspective is only for the rule object recognition, it is necessary to study new methods for identifying 3D geometric structure of arbitrary shape object in the future [19]. This will be a significant breakthrough of 3D object recognition, and the development direction has epoch-making significance.

Fourthly, physical characteristics of the invariance research is a little rare, whether can fuse of other information in image is worth to try and research. It has a profound meaning for combining with other information for 3D recognition.

Fifthly, recognition speed problem is a key issue related to whether it could meet the practical application. Because people has a very high demand for speed and recognition accuracy, there are some contradictions between them, the actual recognition system is both compromise[20,22]. There are two possible solutions, one is using representation method of matching strategy of different levels and model database 
comprehensive to realize the stratified recognition; the other is parallel processing mechanism is effectively introduced to recognition process for realizing stratified recognition.

\section{ACKNOWLEDGEMENTS}

This work was financially supported by Shenyang Science and Technology Foundation (Grant No.F13-316-1-38, 'Research on affine invariant shape recognition based on sub-space’).

\section{REFERENCES}

[1] Qing-Li, ManLi-Zhou, Jian-Liu. Based on multi resolution mesh of 3D object recognition method. Journal of electronics, 2001, 7.

[2] Luoy , Ma H.3D Object Recognition Technique based on Muti-Resolution Aspect Graph Neural Networks and Brain , 2005[A].International Conference[C].2005, 13(15):1168-1172.

[3] YiMin-Cheng, HongXia-Ding, YiXiao-Wang, HaiHong-Zhang. Curved object recognition based on geometrical features China Journal of image and graphics, 2000, 7.

[4] SHEN-CHI TIEN, TSORNG. LIN CHIA, YIBIN LU. Using cross. ratios to model curve data for aircraft recognition[J]. Pattern Recognition Letters, 2003, 24(1 2) : 2047-2060.

[5] JieXian-Ceng, Zhen-Chen. Based on morphological graph representation of a three-dimensional object recognition algorithm. Computer application, 2003, 2.

[6] HaoJun-Tang, Chen-Ming Richard.W Stimets based on layered six angle grid fast recognition of simple 3-D objects within. Journal of computer-aided design \& computer graphics, 2007, 5.

[7] GuoHui-Qin. Based on 3D invariant three-dimensional object recognition. Master degree of Harbin University of Science and Technology, 2009

[8] YING SHAN, HARPREET S. SAWHNEY, BOGDAN MATEI and RAKESHKUMAR. Shapeme histogram projection and matching for partial object recognition[J]. IEEE Trans. Pattern Analysis and Machine Intelligence, 2006, 28(4).
[9] Chen-Tuo, RongChun-Zhao. Geometric invariance and its applications to 3D object recognition. China Journal of image and graphics, 2003, 9.

[10] Jin-Tian, QiangRong-Jiang. Based on multiple view invariance in object recognition. Journal of Beijing University of Technology, 2000, 3.

[11] LiHua-Zou. Three kinds of feature based object recognition algorithm comparison and analysis software guide, 2010, 5.

[12] ZhanZhong-Pang, LiJiao-Cheng, TingYu-Zhang. Based on moment invariant theory and the MATLAB graphic object recognition. Beijing Forestry University, 2009, 8.

[13] THOMAS HESELTINE , PEARS , JIM AUSTIN. Three. dimensional face recognition using combinations of surface feature map subspace components[J]. Image and Vision Computing, 2008, 26(3) : 382-396.

[14] Kai-Song, JianWei-Ji. The chain code form and line segment table in computer image processing applications. Journal of Liaoning Technical University, 2007, 4.

[15] YanJu-Li, Ke-Zhang. The chain code technology in scene image feature extraction. China Journal of image and graphics, 2008.

[16] SHIRAIY . Three-Dimensional Computer Vision[M] . Berlin : Springer-Verlag Berlin Heidelberg, 1987 : 58-66.

[17] EUGENE CHARNIAK, ROBERTP, GOLDMAN. A Bayesian model of plan recognition[J]. Artificial Intelligence, 1993, 64(1) : 53. 79.

[18] FengQi-Zhou, Jian-Sun, Jun-Zhou. A kind of model based affine algebraic invariants. Fire control and command control, 2007.

[19] YiGang-Sun, LiYong-Yang, ChengQi-Sun. The affine coordinate system for multi-view 3D object recognition method. Journal of Civil Aviation University of China, 2005, 12.

[20] V.V.SRINIVAS, SHIVAM, RAO S. GOVINDARAJU. Regional flood frequency analysis by combining self-organizing feature map and fuzzy clustering[J].Journal of Hydrology, 2008, 348(1) : 148-166.

[21] Zhe-Chen, ZeTao-Jiang, ShiZhong-Li, RongChun-Zhao. A new geometric constrained structure and its projective invariant Chinese Journal of computers, 2005, 10.

[22] YiGang-Sun, ChengQi-Sun, YanHong-Du. A 3-D visual recognition method of Chinese civil aviatio 